\title{
Propuesta de principios alternativos para la educación musical en un contexto latinoamericano
}

\section{Proposal of alternative principles for music education in a Latin American context}

Raúl Jorquera Rossel raujorqu@unap.cl

Facultad de Ciencias Humanas

Universidad Arturo Prat Iquique, Chile

ORCID: http://orcid.org/0000-0002-8470-4624

Ximena Valverde Ocariz ximena.valverde@gmail.com Universidad Autónoma de Barcelona ORCID: http://orcid.org/0000-0001-9197-8044

Pere Godall Castel

Pere.Godall@uab.cat

Departamento de Didáctica de la Expresión Musical, Plástica y Corporal Universidad Autónoma de Barcelona

Barcelona, España

ORCID: http://orcid.org/0000-0002-6548-2624

doi: 10.7203/LEEME.46.16932

Recibido: 30-03-2020 Aceptado: 19-05-2020. Contacto y correspondencia: Raúl Jorquera Rossel. Facultad de Ciencias Humanas, Universidad Arturo Prat, Avda. Arturo Prat Chacón, 2120, C.P. 1100000 Iquique. Chile.

\section{Resumen}

Se presenta en este artículo una propuesta de principios educativos alternativos para la educación musical en un contexto latinoamericano. En primer lugar, se describen modelos educativos predominantes que, desde una perspectiva histórica, han determinado el desarrollo de la educación musical en dicho contexto: modelos educativos "jesuita" y "de conservatorio" y paradigma estético-formalista. A continuación, se exponen las principales características del enfoque filosófico praxial, perspectiva teórica que puede ser considerada como una alternativa a las predominancias identificadas. Concluye este trabajo con una propuesta de principios educativos, los que han sido generados desde un contraste teórico entre los modelos detectados y el enfoque filosófico praxial. Esta propuesta busca aportar tanto a futuras investigaciones como a reflexiones pedagógicas, procesos de innovación tanto en currículum como en didácticas, metodologías, formación continua del profesorado y en el diseño y difusión de recursos educativos.

Palabras clave: Modelos educativos predominantes; principios educativos; enfoque filosófico praxial; educación musical.

\section{Abstract}

This article presents a proposal of alternative principles for music education in a Latin American context. First, predominant educational models are described that, from a historical perspective, have determined the development of musical education in this context: "Jesuit" and "conservatory" educational models and an aesthetic-formalist paradigm. Next, the main characteristics of the praxial philosophical approach are exposed, and a theoretical perspective is constituted as an alternative to the identified predominance. This work concludes with a proposal of educational principles, which have been generated from a theoretical contrast between detected models and the praxial philosophical approach. This proposal seeks to contribute to future research as well as for pedagogical reflections, innovation processes in the curriculum as well as in didactics, methodologies, continuous teacher training, and the design and dissemination of educational resources.

Key words: Predominant educational models; educational principles; praxial philosophical approach; music education

@Raúl Jorquera Rossel, Ximena Valverde Ocariz y Pere Godall Castell. The content of this article is the sole responsibility of the authors. The Revista Electrónica de LEEME and Universitat de València are not liable for any legal actions that may arise involving the article's content. Revista Electrónica de LEEME - Lista Electrónica Europea de Música en la Educación-. http://ojs.uv.es/index/php/LEEME/index ISSN: 1575-9563. Editores: Universidad de Valencia y Jesús Tejada. Visibilidad de esta revista: SCOPUS, Emerging Sources Citation Index (Clarivate), EBSCO, CINDOC (CSIC), Citefactor, COPAC, Dialnet, DICE (CSIC), DOAJ, e-revistas (CSIC), EBSCO Premier, ERIH+, Gale Cengage Learning, IN-RECS, IRESIE, LATINDEX, MIAR, OCLC Worldcat, RESH, REDIB, RILM Core Journals, SUDOC, ULRICHS. Esta revista es de acceso libre mediante licencia Creative Commons 4.0 CC by. Política de archivado: etiqueta verde SHERPA-ROMEO. 


\section{Introducción}

En una investigación de mayor alcance (Jorquera, 2019), se analizó la inclusión y exclusión de músicas populares urbanas (González, 2001) en la educación musical chilena y, principalmente, en el ámbito del profesorado de Música (Jorquera y Godall, 2018). Este proceso investigativo propició un incipiente proceso de deconstrucción epistemológica por parte de los investigadores, formados principalmente bajo los parámetros de los modelos y el paradigma a describir. De los resultados de este estudio, se pudo identificar la predominancia que han tenido a lo largo de los años ciertos modelos educativos: los modelos educativos "jesuita" y "de conservatorio" (Shifres y Gonnet, 2015), y el paradigma estético-formalista (Cremades, 2008). Si bien la problemática descrita no es reciente, se considera relevante y pertinente enunciar algunos principios educativos que se constituyan como una alternativa a las premisas de los modelos predominantes identificados. Para este contraste, se consideraron los fundamentos del enfoque filosófico praxial en educación musical expuestos principalmente por Elliott (1995), enfoque que aporta bases argumentales al diseño e implementación de una educación musical formal contextualizada y consistente con los requerimientos educativos del siglo XXI.

\section{La predominancia de modelos educativos musicales}

Los modelos educativos se encuentran en un territorio intermedio entre la práctica docente y los enfoques educativos, y se representan en modelos de formación docente, de enseñanza o de aprendizaje (Jorquera, 2010). Por este motivo, es necesario identificar las características de modelos predominantes en el contexto latinoamericano.

Desde un enfoque crítico del currículum, es fundamental analizar el carácter intercultural de las músicas y la necesidad de desjerarquizar los distintos repertorios, considerando el contexto sociocultural en el que se desarrollan los diversos sistemas educativos (Aróstegui, 2011). Pero, además, se hace necesario, en este sentido, comprender que la educación musical es una disciplina inherentemente interdisciplinar y que, desde esta perspectiva, su contribución al desarrollo educativo se hace aún más imprescindible (Valverde, 2018). Cierto es que a lo largo de la historia ha existido una predominancia de concepciones sobre la música desde una visión eurocentrista, lo que ha tenido como consecuencia la consideración de las músicas como un fenómeno universal y casi homogéneo, desde el prisma de un pensamiento hegeliano (Medivil, 2016). De esta forma, se ha permitido que fuese normal referirse a "la música" en singular, separándose así de toda tradición musical extraeuropea, problemática educativo-musical que es abordada, entre otros, por Polo y Pozzo (2011), quienes sostienen que, en el contexto latinoamericano, las músicas ajenas al canon europeo se insertan en el currículum desde una perspectiva positivista y eurocentrista. Desde esta perspectiva

@Raúl Jorquera Rossel, Ximena Valverde Ocariz y Pere Godall Castell. The content of this article is the sole responsibility of the authors. The Revista Electrónica de LEEME and Universitat de València are not liable for any legal actions that may arise involving the article's content. Revista Electrónica de LEEME - Lista Electrónica Europea de Música en la Educación-. http://ojs.uv.es/index/php/LEEME/index ISSN: 1575-9563. Editores: Universidad de Valencia y Jesús Tejada. Visibilidad de esta revista: SCOPUS, Emerging Sources Citation Index (Clarivate), EBSCO, CINDOC (CSIC), Citefactor, COPAC, Dialnet, DICE (CSIC), DOAJ, e-revistas (CSIC), EBSCO Premier, ERIH+, Gale Cengage Learning, IN-RECS, IRESIE, LATINDEX, MIAR, OCLC Worldcat, RESH, REDIB, RILM Core Journals, SUDOC, ULRICHS. Esta revista es de acceso libre mediante licencia Creative Commons 4.0 CC by. Política de archivado: etiqueta verde SHERPA-ROMEO. 
crítica, es posible identificar la injerencia de unos modelos en este contexto educativo-musical, los que se describen a continuación.

\subsection{Modelo educativo "jesuita"}

Los autores Shifres y Gonnet (2015) identifican y plantean la injerencia del modelo "jesuita" a un modelo de educación musical que formó parte del proceso evangelizador de los conquistadores españoles en Latinoamérica, implantado en reducciones franciscanas, pero, principalmente, en las reducciones administradas por la orden de los jesuitas (Shifres y Gonnet 2015). Este modelo habría sido estructurado desde cuatro características principales, como se puede observar en el siguiente esquema:

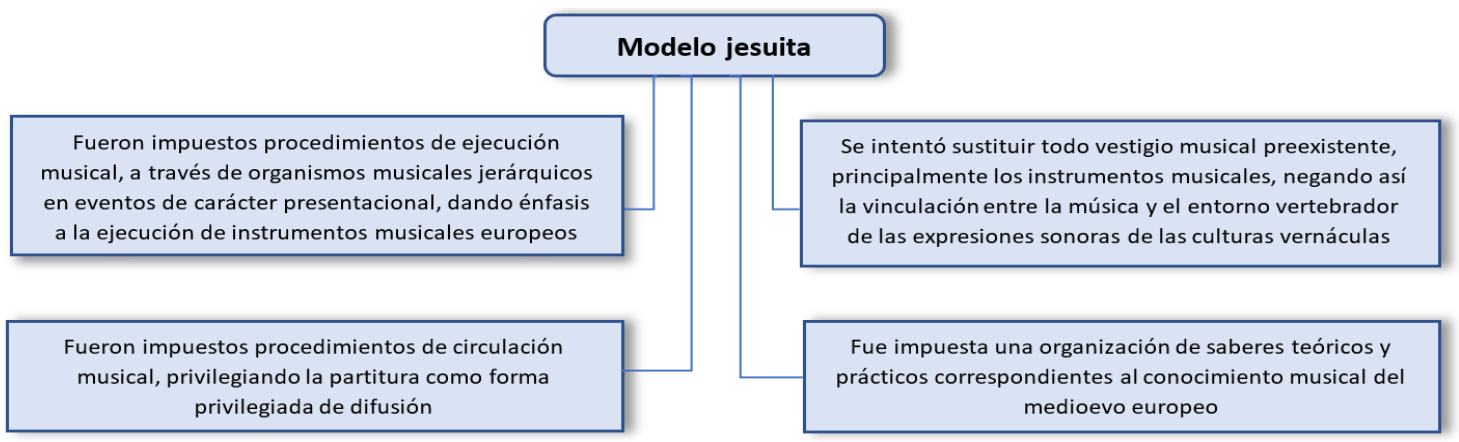

Figura 1. Características principales del modelo educativo "jesuita”. Adaptada de Shifres y Gonnet, 2015, p.55

Este enfoque tuvo como consecuencia el establecer una separación entre quienes tocaban y quienes escuchaban, y donde el saber musical se encontraba en una partitura, que se transformó en el centro del aprendizaje. Además, al ser parte del culto religioso, estableció unos roles interpretativos y representativos, hecho que fue estableciendo un alejamiento de lo que significaba hacer música en forma colectiva, elemento crucial que "es un ejemplo cabal de la disposición espacial devocional que fue adquiriendo el hacer musical a lo largo de los siglos" (Shifres y Gonnet, 2015, p.56).

Toda esta concepción incluyó tensiones de tipo racial, de libertad, de cosmovisión sonora, de temporalidades, que se mantienen aún vivas en la actualidad (Shifres y Gonet, 2015). Así, la imposición de este modelo tuvo las siguientes consecuencias:

- Supresión ontológica de la música y del sujeto músico de los pueblos que habitaban el continente.

- Imposición de la cultura musical alfabetizada como sinónimo de saber musical.

- Al posibilitar el desarrollo de formas discursivas complejas que no se hallan necesariamente supeditadas a las restricciones de la memoria, melló también el

@Raúl Jorquera Rossel, Ximena Valverde Ocariz y Pere Godall Castell. The content of this article is the sole responsibility of the authors. The Revista Electrónica de LEEME and Universitat de València are not liable for any legal actions that may arise involving the article's content. Revista Electrónica de LEEME - Lista Electrónica Europea de Música en la Educación-. http://ojs.uv.es/index/php/LEEME/index ISSN: 1575-9563. Editores: Universidad de Valencia y Jesús Tejada. Visibilidad de esta revista: SCOPUS, Emerging Sources Citation Index (Clarivate), EBSCO, CINDOC (CSIC), Citefactor, COPAC, Dialnet, DICE (CSIC), DOAJ, e-revistas (CSIC), EBSCO Premier, ERIH+, Gale Cengage Learning, IN-RECS, IRESIE, LATINDEX, MIAR, OCLC Worldcat, RESH, REDIB, RILM Core Journals, SUDOC, ULRICHS. Esta revista es de acceso libre mediante licencia Creative Commons 4.0 CC by. Política de archivado: etiqueta verde SHERPA-ROMEO. 
lugar de privilegio que tenían las formas más simples (en general, binarias y repetitivas).

- Estableció una brecha inexistente antes entre músicos (alfabetizados, que piensan en esos términos y de acuerdo con esas categorías) y no músicos (no alfabetizados, que entienden la música en términos no notacionales).

- Estableció la brecha entre qué es pensar musicalmente y qué es escuchar o incluso tocar música, sin pensar musicalmente, marcando el límite entre lo musical y lo extramusical (Shifres y Gonnet, 2015).

\subsection{Modelo educativo "de conservatorio"}

Shifres y Gonnet (2015) indentifican y describen, además, la predominancia de otro modelo educativo que denominan modelo "de conservatorio". El proceso histórico que dio como resultado la implantación de este modelo es vinculado por los autores al relevo de una cosmovisión católica por una protestante en el siglo XVII vinculado a la problemática colonial. Esta circunstancia "trajo aparejados cambios importantes en las concepciones acerca del arte y de la formación de los artistas" (Shifres y Gonnet, 2015, p.57), situándose como factor clave en la instauración de este modelo en Latinoamérica la creación del Conservatorio de París a finales del siglo XVIII. La influencia de esta institución, primero en el resto de Europa y después en América, se sumó en este continente a la influencia que continuaba teniendo allí el modelo jesuita, superponiendo a este gradualmente otras características que son descritas por los autores de la siguiente manera:

\section{Modelo de conservatorio}

Separación entre el músico y el auditor, representada en el formato del "concierto"

Encuadre diádico. Relación pedagógica basada en la interacción entre un maestro y un discípulo
Secularización de la educación musical

Separación entre la figura del compository el ejecutante

Valoración de la formación y de los desempeños individuales. El conservatorio forma instrumentistas para ser solistas o miembros de orquestas o grupos de cámara

Figura 2. Características principales del modelo educativo de conservatorio. Adaptada de Shifres y Gonnet, 2015, pp.58-62

El principal impacto que este modelo educativo ha tenido en la educación musical latinoamericana es asignar como un fin de la educación musical el centrar el aprendizaje en la partitura, oficializando métodos de aprendizaje donde el texto musical es el eje central, conservado por un cuerpo de maestros. Esto tuvo como consecuencia que "el conservatorio extiende la colonialidad del saber musical a un plano ontológico, determinando que la música es

@Raúl Jorquera Rossel, Ximena Valverde Ocariz y Pere Godall Castell. The content of this article is the sole responsibility of the authors. The Revista Electrónica de LEEME and Universitat de València are not liable for any legal actions that may arise involving the article's content. Revista Electrónica de LEEME - Lista Electrónica Europea de Música en la Educación-. http://ojs.uv.es/index/php/LEEME/index ISSN: 1575-9563. Editores: Universidad de Valencia y Jesús Tejada. Visibilidad de esta revista: SCOPUS, Emerging Sources Citation Index (Clarivate), EBSCO, CINDOC (CSIC), Citefactor, COPAC, Dialnet, DICE (CSIC), DOAJ, e-revistas (CSIC), EBSCO Premier, ERIH+, Gale Cengage Learning, IN-RECS, IRESIE, LATINDEX, MIAR, OCLC Worldcat, RESH, REDIB, RILM Core Journals, SUDOC, ULRICHS. Esta revista es de acceso libre mediante licencia Creative Commons 4.0 CC by. Política de archivado: etiqueta verde SHERPA-ROMEO. 
el texto, es decir, el conjunto de marcas en la partitura, y el músico es el que conoce el texto" (Shifres y Gonnet, 2015, p.60).

Esta perspectiva ha tenido un fuerte impacto en los procesos educativos a nivel latinoamericano en diversos niveles: en la formación del profesorado, en la estructuración de las políticas educativas, en la práctica educativa de los docentes y, a su vez, una fuerte injerencia en el rechazo a las músicas no vinculadas a la tradición escrita, dando espacios ínfimos a las músicas pertenecientes a la otra tradición: la tradición oral. La injerencia que hasta el día de hoy ha tenido este modelo queda muy bien resumida por ambos autores al decir que la educación musical tendría, bajo las premisas de este, dos claros objetivos: por un lado, intentar formar músicos que dominen el lenguaje escrito en ámbitos especializados; por otro lado, formar oyentes, receptivos a la labor de los otros (Shifres y Gonnet, 2015).

No está de más reflexionar en este punto que tanto el llamado modelo "jesuita" como, así mismo, el modelo "de conservatorio" cuentan con características propias que, observadas desde una perspectiva de las teorías del aprendizaje, se asemejan a teorías de carácter conductista. De modo que, el aprendizaje se consigue a partir de la repetición y, la mayoría de las veces está exento de un proceso de reflexión, por lo que dicho proceso carece de significado para el individuo involucrado (Valverde, 2018).

\subsection{Paradigma estético-formalista}

En conjunto con los modelos descritos, en la educación musical formal existe aún una fuerte predominancia de un paradigma estético-formalista, caracterizado por "un tipo de pensamiento propio de la modernidad, que somete a la música y al arte en general a un proceso de racionalización que deviene en una separación entre vida y música” (Cremades, 2008, p.5). Este paradigma educativo tendría sus orígenes en que los pensamientos de "filósofos musicales que van de 1750 a 1950 tienen asumido a priori que es natural concebir y percibir la música en términos de atributos auto-referenciales, absolutos y universales" (Cremades, 2008, p.5).

Este enfoque se vincula, directamente a su vez, con las denominadas "bellas artes", concepto que ha dominado la concepción artística de las músicas y que ha tenido una fuerte presencia en el ámbito educativo, tal como expone Romé (2015). La formación musical en Latinoamérica tiene una fuerte injerencia paradigmática desde la esfera de lo que se entiende por Bellas Artes, cuyas estéticas hegemónicas se explicarían en: a) una tendencia a buscar la trascendencia de esta artes a cualquier carácter o funcional-social, b) un énfasis en el desarrollo de competencias técnico-instrumentales, c) la relevancia al elemento formal de las obras desde su perspectiva compositiva, d) la búsqueda de universalidad de la música y e) una separación de roles entre intérprete y compositor (Romé, 2015, p.7).

@Raúl Jorquera Rossel, Ximena Valverde Ocariz y Pere Godall Castell. The content of this article is the sole responsibility of the authors. The Revista Electrónica de LEEME and Universitat de València are not liable for any legal actions that may arise involving the article's content. Revista Electrónica de LEEME - Lista Electrónica Europea de Música en la Educación-. http://ojs.uv.es/index/php/LEEME/index ISSN: 1575-9563. Editores: Universidad de Valencia y Jesús Tejada. Visibilidad de esta revista: SCOPUS, Emerging Sources Citation Index (Clarivate), EBSCO, CINDOC (CSIC), Citefactor, COPAC, Dialnet, DICE (CSIC), DOAJ, e-revistas (CSIC), EBSCO Premier, ERIH+, Gale Cengage Learning, IN-RECS, IRESIE, LATINDEX, MIAR, OCLC Worldcat, RESH, REDIB, RILM Core Journals, SUDOC, ULRICHS. Esta revista es de acceso libre mediante licencia Creative Commons 4.0 CC by. Política de archivado: etiqueta verde SHERPA-ROMEO. 
A continuación, se exponen las características de este paradigma:

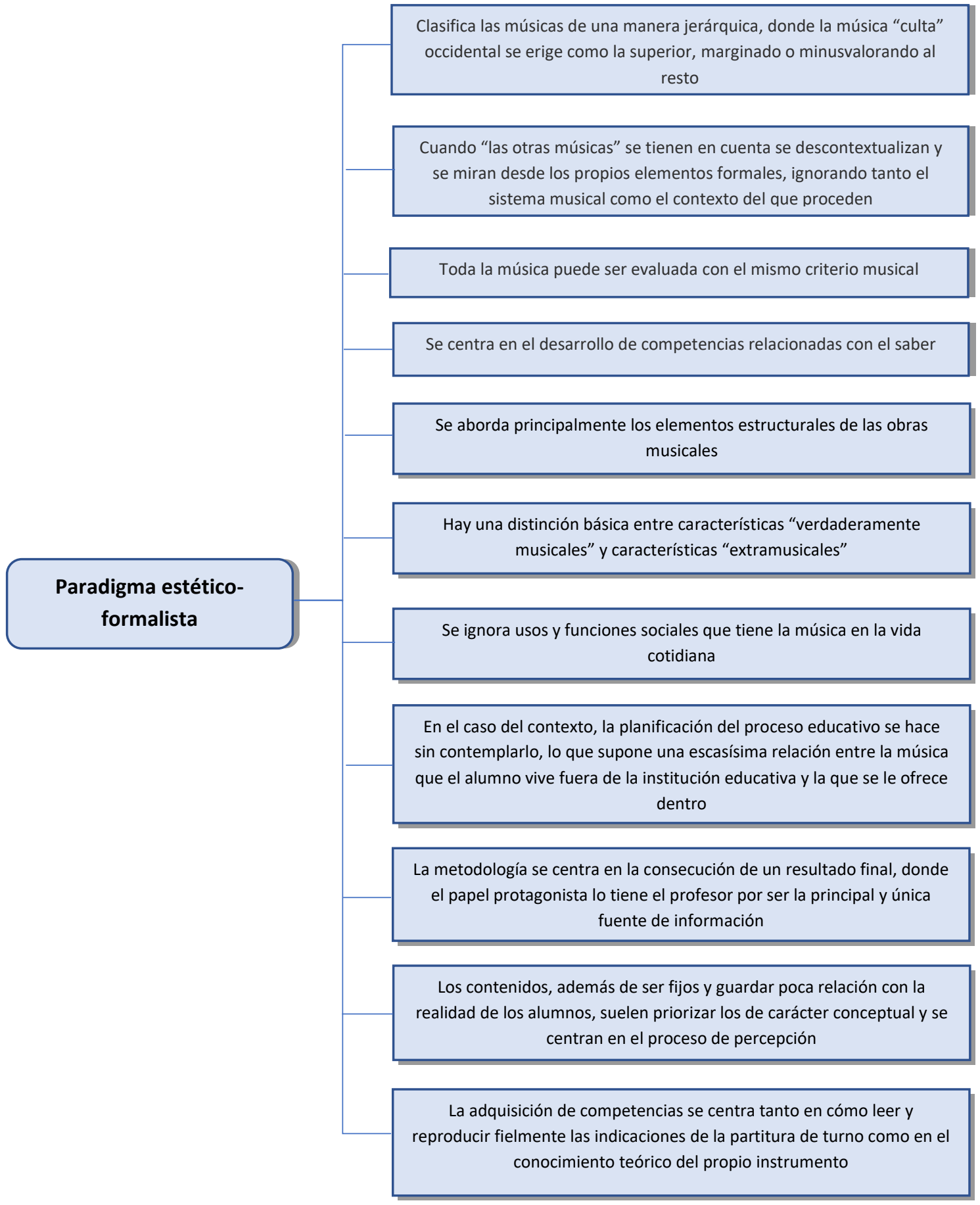

Figura 3. Principales características del paradigma estético-formalista. Adaptada de Cremades, 2008, p.6

@Raúl Jorquera Rossel, Ximena Valverde Ocariz y Pere Godall Castell. The content of this article is the sole responsibility of the authors. The Revista Electrónica de LEEME and Universitat de València are not liable for any legal actions that may arise involving the article's content. Revista Electrónica de LEEME - Lista Electrónica Europea de Música en la Educación-. http://ojs.uv.es/index/php/LEEME/index ISSN: 1575-9563. Editores: Universidad de Valencia y Jesús Tejada. Visibilidad de esta revista: SCOPUS, Emerging Sources Citation Index (Clarivate), EBSCO, CINDOC (CSIC), Citefactor, COPAC, Dialnet, DICE (CSIC), DOAJ, e-revistas (CSIC), EBSCO Premier, ERIH+, Gale Cengage Learning, IN-RECS, IRESIE, LATINDEX, MIAR, OCLC Worldcat, RESH, REDIB, RILM Core Journals, SUDOC, ULRICHS. Esta revista es de acceso libre mediante licencia Creative Commons 4.0 CC by. Política de archivado: etiqueta verde SHERPA-ROMEO. 


\section{Enfoque filosófico praxial}

Para efectos de esta propuesta, se ha seleccionado este enfoque al tener una perspectiva que permite la generación de un contraste teórico con las premisas de los modelos y el paradigma descrito, desde una visión integral, interdisciplinar y, por qué no reconocerlo, también por su enfoque funcional, que puede ser replicable a otros contextos y realidades educativo-musicales.

Murillo (2015) propone un acercamiento al enfoque filosófico praxial de David Elliott, al "plantear el aprendizaje musical desde un enfoque pragmatista (...) implica trabajar desde la misma acción y contemplar la creación sonora como un elemento necesario para garantizar una mejora en las prácticas habituales en el aula" (Murillo, 2015, p.49). Las propuestas educativas de Elliott (1995) se basan en el pensamiento de John Dewey, quien en su obra "El Arte como experiencia" (1934), sienta las bases del alejamiento de la Educación Artística de las premisas ligadas a lo estético-formalista, argumentando que el soporte de transmisión del Arte no se desarrolla en contextos de objetividad ni de subjetividad, y que lo que le otorga su dimensión artística es, precisamente, el entrar en contacto con los individuos a través de experiencias únicas (Dewey, 2008).

De esta forma, su legado fue adaptado posteriormente al ámbito de la didáctica de la educación musical por Elliott (1995) que consolida el enfoque filosófico praxial, concepto que no ha estado exento de problemas de traducción del inglés al castellano, tal como explica Pliego (2012): algunos autores le han denominado filosofía o paradigma "pragmatista" y en algunas publicaciones como "práctico" (Pliego, 2012). Para efectos de este trabajo, se considerará su postura y se le denominará enfoque filosófico praxial. En su enfoque, el alejamiento del enfoque estético-formalista ya propuesto por Dewey (1934) es continuado por Elliott (1995), llevando estas reflexiones al plano de lo musical. Así, se sostiene que la finalidad de lograr "experiencias estéticas" ha potenciado en la educación musical un solo tipo de acercamiento al hecho musical, que se basaría en centrarse "exclusivamente en sus cualidades estructurales o estéticas" (Elliott, 1995, p.22).

El mismo autor describe las dos características principales del enfoque filosófico praxial en educación musical: la primera, que la naturaleza de la educación musical depende de las concepciones que se tengan de lo que es la música, y la segunda, que el significado de la educación musical dependerá de la relevancia y el significado que se le dé a la música en la vida cotidiana (Elliott, 1995). En esta línea, Rosabal-Coto (2008) subraya que la educación musical debería dar relevancia al significado de las músicas en la vida cotidiana de las personas para evitar ser poco significativa en una proyección futura, abogando por una apertura al significado que las personas dan a sus músicas y al uso que le dan en sus vidas, porque, de no ser así, "no será más que una praxis estéril" (Rosabal-Coto, 2008, p.15). Esta argumentación está en

@Raúl Jorquera Rossel, Ximena Valverde Ocariz y Pere Godall Castell. The content of this article is the sole responsibility of the authors. The Revista Electrónica de LEEME and Universitat de València are not liable for any legal actions that may arise involving the article's content. Revista Electrónica de LEEME - Lista Electrónica Europea de Música en la Educación-. http://ojs.uv.es/index/php/LEEME/index ISSN: 1575-9563. Editores: Universidad de Valencia y Jesús Tejada. Visibilidad de esta revista: SCOPUS, Emerging Sources Citation Index (Clarivate), EBSCO, CINDOC (CSIC), Citefactor, COPAC, Dialnet, DICE (CSIC), DOAJ, e-revistas (CSIC), EBSCO Premier, ERIH+, Gale Cengage Learning, IN-RECS, IRESIE, LATINDEX, MIAR, OCLC Worldcat, RESH, REDIB, RILM Core Journals, SUDOC, ULRICHS. Esta revista es de acceso libre mediante licencia Creative Commons 4.0 CC by. Política de archivado: etiqueta verde SHERPA-ROMEO. 
sintonía con Regelski (2009), quien evidencia la problemática de situar el repertorio trabajado en el aula en extremos tan distantes de la realidad que el estudiantado vive en contextos extraaula y como consecuencia, que esta desvinculación genera tensiones con su vida futura.

En la línea de dar relevancia al impacto a largo plazo de la educación musical (Regelski, 2009), Herrada (2016, p.207) ofrece una clarificadora reflexión vinculada a la relevancia de dicha utilidad, al afirmar que la educación formal debería orientarse a lograr una validez del conocimiento, a que este conocimiento sea útil y que "genere respuestas y soluciones inmediatas a las necesidades del individuo en formación”. También, en concordancia con McLaren (1990), quien propone que el conocimiento que se aborde en el aula debe ser relevante para el estudiantado, proponiendo que debiera existir una mayor participación de los jóvenes en las planificaciones lectivas de los docentes como parte de una retroalimentación pedagógica. Esta premisa de la retroalimentación pedagógica que se debería lograr entre discentes y docentes, como individuos que comparten un contexto similar, es reforzada a su vez por Macdonald (2016), quien expone y respalda que el enfoque filosófico praxial es la alternativa para fusionar los intereses de los docentes con los de los estudiantes.

Del mismo modo, la consideración de los contextos y de las realidades sonoras extraaula con las que convive el estudiantado ha sido también abordada por Arredondo y García (1998, p.92), quienes argumentan que, desde este enfoque de carácter praxial, se deben reconocer estos contextos, utilizándolos como eje de la formación "desde valores y actitudes críticas, solidarias y tolerantes".

En sintonía con estas reflexiones, Froehlich (2011) dice que cuando en la educación musical formal no se reconocen los vínculos sonoros del estudiantado con sus familiares o con sus pares, se corre el peligro de llegar solo a una forma de escolarización, y no a una plena educación. Continúa afirmando que, si se considera que la relevancia del conocimiento tiene una connotación subjetiva, el que los contextos sonoros reales de los estudiantes sean distintos a los que se trabajan en el aula produce conflictos relacionados con la importancia de lo que se aprende en la educación musical formal (Froehlich, 2011).

Por otra parte, en el contexto chileno, Uribe (2010) sostiene que el impacto de las músicas actuales es tan fuerte que el profesorado se ha visto en la obligación de cambiar, no solo las estructuras de legitimación y jerarquización, sino, además, en la praxis, las antiguas metodologías que ahora no alcanzan a dar cobertura a las necesidades del estudiantado. Esta circunstancia lleva a que las estrategias anteriores pierdan su carga simbólica. El mismo autor conecta además con la idea de la utilidad futura de la educación musical, ya expuesta con anterioridad, pero agregando un elemento distinto: que ya no basta solo con comprender el contexto, sino también que, desde una perspectiva crítica, es posible incluso llegar a cambiarlo (Uribe, 2010, p.117).

@Raúl Jorquera Rossel, Ximena Valverde Ocariz y Pere Godall Castell. The content of this article is the sole responsibility of the authors. The Revista Electrónica de LEEME and Universitat de València are not liable for any legal actions that may arise involving the article's content. Revista Electrónica de LEEME - Lista Electrónica Europea de Música en la Educación-. http://ojs.uv.es/index/php/LEEME/index ISSN: 1575-9563. Editores: Universidad de Valencia y Jesús Tejada. Visibilidad de esta revista: SCOPUS, Emerging Sources Citation Index (Clarivate), EBSCO, CINDOC (CSIC), Citefactor, COPAC, Dialnet, DICE (CSIC), DOAJ, e-revistas (CSIC), EBSCO Premier, ERIH+, Gale Cengage Learning, IN-RECS, IRESIE, LATINDEX, MIAR, OCLC Worldcat, RESH, REDIB, RILM Core Journals, SUDOC, ULRICHS. Esta revista es de acceso libre mediante licencia Creative Commons 4.0 CC by. Política de archivado: etiqueta verde SHERPA-ROMEO. 
A continuación, se presentan las principales características de este enfoque:

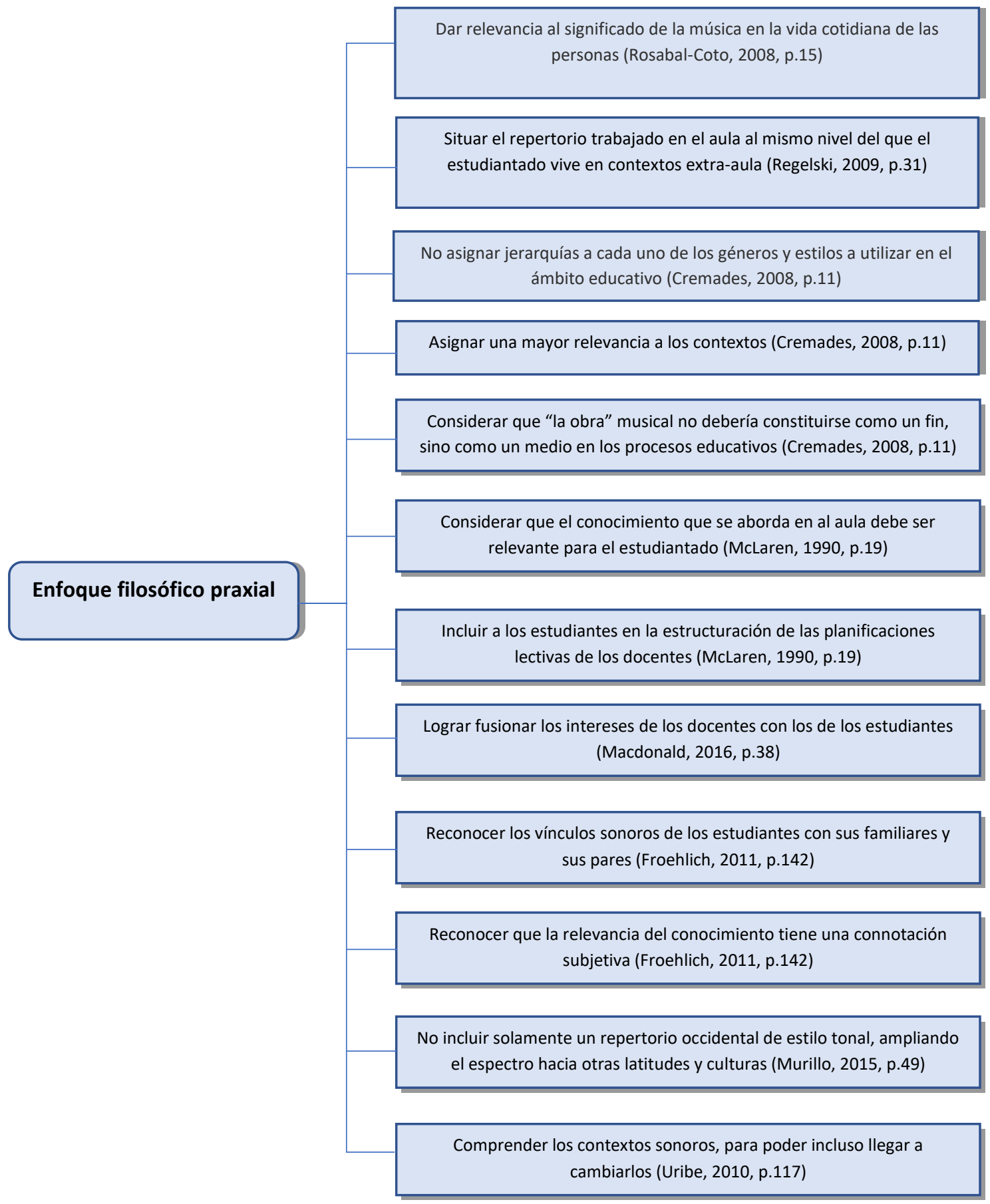

Figura 4. Características principales del enfoque filosófico praxial. Fuente: Jorquera, 2019, p.46

@Raúl Jorquera Rossel, Ximena Valverde Ocariz y Pere Godall Castell. The content of this article is the sole responsibility of the authors. The Revista Electrónica de LEEME and Universitat de València are not liable for any legal actions that may arise involving the article's content. Revista Electrónica de LEEME - Lista Electrónica Europea de Música en la Educación-. http://ojs.uv.es/index/php/LEEME/index ISSN: 1575-9563. Editores: Universidad de Valencia y Jesús Tejada. Visibilidad de esta revista: SCOPUS, Emerging Sources Citation Index (Clarivate), EBSCO, CINDOC (CSIC), Citefactor, COPAC, Dialnet, DICE (CSIC), DOAJ, e-revistas (CSIC), EBSCO Premier, ERIH+, Gale Cengage Learning, IN-RECS, IRESIE, LATINDEX, MIAR, OCLC Worldcat, RESH, REDIB, RILM Core Journals, SUDOC, ULRICHS. Esta revista es de acceso libre mediante licencia Creative Commons 4.0 CC by. Política de archivado: etiqueta verde SHERPA-ROMEO. 


\section{Propuesta de principios educativos alternativos}

Esta propuesta nace con el objetivo de aportar teóricamente alineamientos curriculares, tanto en los diversos niveles educativo-musicales como, a su vez, en la formación docente de la especialidad en el contexto latinoamericano. Emerge desde el contraste teórico de los enunciados del paradigma estético praxial con las premisas de los modelos "jesuita", "de conservatorio" y el paradigma estético-formalista. Al existir ciertos puntos de encuentro entre los modelos "jesuita" y "de conservatorio" y el paradigma estético-formalista, algunos principios propuestos como alternativa a cada uno de estos enfoques presentan similitudes en sus enunciados.

\subsection{Principios educativos alternativos al modelo educativo "jesuita"}

- Revaloración de los vestigios musicales precolombinos, sobre todo desde la inclusión de especialidades como la arqueo-musicología en la formación del profesorado, intentando siempre valorar estas expresiones sonoras de las culturas originarias desde espacios alejados de unas consideraciones puramente estéticas y desde las dimensiones que entienden la música única y exclusivamente como "Arte".

- Recuperación de saberes teóricos y prácticos de las culturas originarias del continente, reconociendo la presencia de unos saberes correspondientes al conocimiento musical europeo, pero siempre contextualizándolos dentro de la problemática de la imposición cultural producto del colonialismo.

- Asignación de un mayor énfasis al desarrollo de un aprendizaje práctico-oral en todas las dimensiones de la educación musical, sin dar excesiva relevancia al "código" representado por la partitura, considerando que el medio de creación y transmisión de la mayoría de las músicas presentes en los contextos extraaula de los estudiantes no se relaciona con la dimensión escrita de las músicas.

- Asignación de una mayor relevancia a los procesos educativos por sobre los énfasis de los resultados, vinculados a eventos escenificados. Desjerarquización de las estructuras en las que se comparte el hecho musical en el interior del aula. Inclusión de instrumentos de las más diversas procedencias a los que se tenga acceso.

- Revaloración de formas simples musicales. Evitar la tendencia a asociar la calidad en la educación con la complejidad de los repertorios a abordar, elemento que permitiría potenciar la memoria en el aprendizaje musical desde la imitación y la repetición.

- Ampliación del espectro de participación en actividades musicales de aula a todos los estudiantes, desde las características, intereses y necesidades de cada

@Raúl Jorquera Rossel, Ximena Valverde Ocariz y Pere Godall Castell. The content of this article is the sole responsibility of the authors. The Revista Electrónica de LEEME and Universitat de València are not liable for any legal actions that may arise involving the article's content. Revista Electrónica de LEEME - Lista Electrónica Europea de Música en la Educación-. http://ojs.uv.es/index/php/LEEME/index ISSN: 1575-9563. Editores: Universidad de Valencia y Jesús Tejada. Visibilidad de esta revista: SCOPUS, Emerging Sources Citation Index (Clarivate), EBSCO, CINDOC (CSIC), Citefactor, COPAC, Dialnet, DICE (CSIC), DOAJ, e-revistas (CSIC), EBSCO Premier, ERIH+, Gale Cengage Learning, IN-RECS, IRESIE, LATINDEX, MIAR, OCLC Worldcat, RESH, REDIB, RILM Core Journals, SUDOC, ULRICHS. Esta revista es de acceso libre mediante licencia Creative Commons 4.0 CC by. Política de archivado: etiqueta verde SHERPA-ROMEO. 
uno, factor que permitiría a largo plazo generar la imagen de que una gran mayoría de la población puede participar en forma activa en iniciativas musicales, desarraigando la idea de que la música es algo "difícil" de hacer.

- Ampliación de las barreras del hecho musical, no circunscribirlo a las características internas de las músicas. Incluir elementos del musicking de Small (1998), entendiendo que todos los involucrados en el proceso que da sentido a cualquier hecho musical son elementos constituyentes del mismo.

\subsection{Principios educativos alternativos al modelo educativo "de conservatorio"}

- Consideración de otros tipos de relaciones en el interior del aula. Las nuevas teorías educativas han constituido al docente más como un "mediador" que como un "maestro", motivo por el que sería relevante incluir el aprendizaje colaborativo entre pares e importar al aula formatos de aprendizaje informal de las músicas.

- Valoración de los desempeños colectivos, dando más énfasis a los procesos que a los resultados y asignando objetivos de superación mayormente asequibles a todos los estudiantes participantes de cualquier actividad musical, independientemente de sus habilidades.

- Inclusión de la creatividad como eje central de la educación musical. La creatividad debería ser un eje en la formación del docente, desvinculándola de la tendencia a relacionarla solo con la composición de "obras" o con un tipo de improvisación asociada al jazz. Desarrollar la creatividad desde la simplicidad de una sola nota ya sería un ejercicio que podría lograr que los estudiantes internalizaran y luego replicaran "acciones creativas".

- Relativización del formato del concierto como único medio de canalización de acciones musicales. Revaloración del hecho musical como un acto colectivo, participativo, inclusivo y des-jerarquizado.

- Asignación de una alta importancia al uso y funciones que dan los estudiantes a las músicas de su preferencia en contextos extra-aula.

- Relativización de la relevancia dada a los componentes internos de las músicas y a la inclinación a considerar todo lo ajeno a estos componentes como elementos "extramusicales".

- Relativización de la tendencia a considerar la música como un elemento universal, para entenderla más bien como un constructo socio-cultural que da soporte a la coexistencia de muchas músicas, cada una de ellas con unas características, fines, usos y significados particulares.

- Des-jerarquización de los distintos repertorios musicales, dando importancia a los contextos en los que se desarrollan y a sus características, fines, usos y

@Raúl Jorquera Rossel, Ximena Valverde Ocariz y Pere Godall Castell. The content of this article is the sole responsibility of the authors. The Revista Electrónica de LEEME and Universitat de València are not liable for any legal actions that may arise involving the article's content. Revista Electrónica de LEEME - Lista Electrónica Europea de Música en la Educación-. http://ojs.uv.es/index/php/LEEME/index ISSN: 1575-9563. Editores: Universidad de Valencia y Jesús Tejada. Visibilidad de esta revista: SCOPUS, Emerging Sources Citation Index (Clarivate), EBSCO, CINDOC (CSIC), Citefactor, COPAC, Dialnet, DICE (CSIC), DOAJ, e-revistas (CSIC), EBSCO Premier, ERIH+, Gale Cengage Learning, IN-RECS, IRESIE, LATINDEX, MIAR, OCLC Worldcat, RESH, REDIB, RILM Core Journals, SUDOC, ULRICHS. Esta revista es de acceso libre mediante licencia Creative Commons 4.0 CC by. Política de archivado: etiqueta verde SHERPA-ROMEO. 
significados particulares de cada uno de ellos y ampliando la mirada hacia músicas de otras culturas, dando valor al conocimiento y comprensión del mundo desde la educación musical.

- Inclusión de todas las músicas, dando relevancia a sus contextos de origen, no estableciendo única y exclusivamente valoraciones asociadas a los componentes internos de las mismas.

\subsection{Principios educativos alternativos al paradigma estético-formalista}

- Relativización de la jerarquía que usualmente se da a los elementos estructurales de las músicas para dar paso a una valoración del hecho musical en su integralidad, en su relación con los contextos e intereses de todos los involucrados en los procesos de creación, producción, difusión y recepción.

- Relativización de la jerarquía que usualmente se da al componente cognitivo y, en menor grado, al componente procedimental en la educación musical formal, para dar paso a una inclusión y valoración de un ámbito que es fundamental en todo lo relacionado al hecho musical: el ámbito emocional.

- Relativización de la jerarquía que usualmente se da al desarrollo de competencias asociadas a la lectoescritura musical y al desarrollo de las técnicas instrumentales. Si bien estas acciones son parte de los procesos educativos en el ámbito de la educación musical, no deberían constituirse como un fin en sí mismo, sino más bien como un medio que permita el acceso al hecho musical a estudiantes que no consideren entre sus necesidades educativas el desarrollo de tales competencias.

- Instauración de planificaciones flexibles y participativas generadas desde las propias necesidades e intereses de los estudiantes y de las pautas que el docente estime como útiles para la relación de sus estudiantes con el mundo sonoro que les rodea en contextos extra-aula.

- Instauración de metodologías centradas en los procesos más que en los resultados, asignando objetivos de superación diferenciados, independientemente de las habilidades de los estudiantes. A su vez, el profesor no es la única fuente de información, por lo que es relevante incluir el aprendizaje colaborativo entre pares, el autodidactismo e importar al aula formatos de aprendizaje informal de las músicas.

- Asignación de una alta relevancia a los contextos, tanto a los que dan sentido a las músicas que se abordan en los procesos educativos, como a los contextos sonoros extra-aula en los que se desenvuelven los estudiantes.

@Raúl Jorquera Rossel, Ximena Valverde Ocariz y Pere Godall Castell. The content of this article is the sole responsibility of the authors. The Revista Electrónica de LEEME and Universitat de València are not liable for any legal actions that may arise involving the article's content. Revista Electrónica de LEEME - Lista Electrónica Europea de Música en la Educación-. http://ojs.uv.es/index/php/LEEME/index ISSN: 1575-9563. Editores: Universidad de Valencia y Jesús Tejada. Visibilidad de esta revista: SCOPUS, Emerging Sources Citation Index (Clarivate), EBSCO, CINDOC (CSIC), Citefactor, COPAC, Dialnet, DICE (CSIC), DOAJ, e-revistas (CSIC), EBSCO Premier, ERIH+, Gale Cengage Learning, IN-RECS, IRESIE, LATINDEX, MIAR, OCLC Worldcat, RESH, REDIB, RILM Core Journals, SUDOC, ULRICHS. Esta revista es de acceso libre mediante licencia Creative Commons 4.0 CC by. Política de archivado: etiqueta verde SHERPA-ROMEO. 


\section{Reflexiones a modo de cierre}

Las premisas del enfoque filosófico praxial se posicionan como una alternativa epistemológica relevante frente a las predominancias descritas. De modo que establecer unos principios educativos desde esta perspectiva, a modo de contraste con las premisas de los modelos descritos, permitiría dar continuidad a una línea investigativa orientada a concretar la aplicabilidad de los mencionados principios. Esta línea de investigación otorgaría orientaciones para la conformación de modelos educativos y estructuras curriculares concretadas en planes de estudio (tanto para políticas educativas oficiales como para la formación docente). Al mismo tiempo, potenciaría el desarrollo de metodologías y estrategias didácticas específicas y contextualizadas a diferentes espacios y realidades sociales y culturales, reconociendo en ellas sus costumbres, tradiciones y creencias específicas.

Al observar cada uno de los tres apartados de la propuesta, es posible identificar una serie de ejes transversales:

a) Revaloración de los vestigios musicales precolombinos, de la diversidad de contexto y de los desempeños colectivos, participativos e inclusivos.

b) Equilibrio entre lo práctico-oral y el código representado por la partitura.

c) Des-jerarquización de repertorios y de estructuras al interior de aula.

d) Revaloración de formas musicales simples.

e) Mayor inclusión de intereses y necesidades del estudiantado, instaurando planificaciones flexibles y participativas.

f) Potenciación de la creatividad.

g) Alejamiento de una mirada universalista de la música, ampliando la mirada hacia músicas de otras culturas.

h) Dar énfasis al conocimiento y comprensión del mundo desde la educación musical.

i) Búsqueda de un mayor equilibrio ente los componentes cognitivos y emocionales.

Cada uno de estos ejes se presentan como alternativas que, según la percepción aquí presentada, podrían aportar no solo a futuras investigaciones, sino también a reflexiones pedagógicas, procesos de innovación en currículum, didácticas y metodologías, formación continua del profesorado y al diseño y difusión de recursos educativos.

$\mathrm{Si}$ bien en este trabajo fueron identificados modelos educativos predominantes de carácter eurocéntrico, no serían las únicas injerencias que determinan el desarrollo de la educación musical en el contexto latinoamericano. Al mismo tiempo, las premisas del enfoque filosófico praxial en ningún caso pueden considerarse como único referente teórico desde el cuál se pueden generar alternativas a los modelos descritos. Así pues, sería altamente relevante

@Raúl Jorquera Rossel, Ximena Valverde Ocariz y Pere Godall Castell. The content of this article is the sole responsibility of the authors. The Revista Electrónica de LEEME and Universitat de València are not liable for any legal actions that may arise involving the article's content. Revista Electrónica de LEEME - Lista Electrónica Europea de Música en la Educación-. http://ojs.uv.es/index/php/LEEME/index ISSN: 1575-9563. Editores: Universidad de Valencia y Jesús Tejada. Visibilidad de esta revista: SCOPUS, Emerging Sources Citation Index (Clarivate), EBSCO, CINDOC (CSIC), Citefactor, COPAC, Dialnet, DICE (CSIC), DOAJ, e-revistas (CSIC), EBSCO Premier, ERIH+, Gale Cengage Learning, IN-RECS, IRESIE, LATINDEX, MIAR, OCLC Worldcat, RESH, REDIB, RILM Core Journals, SUDOC, ULRICHS. Esta revista es de acceso libre mediante licencia Creative Commons 4.0 CC by. Política de archivado: etiqueta verde SHERPA-ROMEO. 
ampliar y enriquecer los alcances teóricos de esta propuesta desde otros enfoques epistemológicos con los que existen posiciones similares frente a la problemática abordada, tales como el campo de la ideología y el poder, las hegemonías educativas, el currículum crítico, las teorías decoloniales, los enfoques de género, entre otros.

En concordancia con lo anterior, consideramos que se hace necesario ampliar los alcances de este estudio hacia todos aquellos contextos educativos en los que existan educadores que sientan la necesidad de cuestionar los parámetros establecidos. Esta ampliación permitiría además dar énfasis en el qué, para qué y cómo se incluyen las músicas en los más diversos espacios educativos formales. Para concluir, creemos que es de especial importancia buscar nuevos caminos para que la educación musical pueda tener un sentido reflexivo, contribuyendo así al desarrollo de un pensamiento libre, crítico y autónomo.

\section{Referencias}

Aróstegui, J. (2011). Por un currículum contrahegemónico: de la educación musical a la música educativa. Revista Da ABEM. V. 19(5), 19-29. Recuperado de: http://abemeducacaomusical.com.br/revista_abem/ed25/revista25_artigo2.pdf

Arredondo, H. y García, F. (1998). Música popular moderna en la formación del profesorado. Eufonía. Didáctica de la Música, 12, 91-98. Recuperado de: http://chile.grao.com/es/producto/musica-popular-moderna-en-la-formacion-delprofesorado

Cremades, A. (2008). El pragmatismo y las competencias en educación musical. Revista Electrónica de LEEME, 21, 1-16. Recuperado de: https://ojs.uv.es/index.php/LEEME/article/view/9769/9203

Dewey, J. (2008). El arte como experiencia. Barcelona: Paidós Ibérica, S.A. Recuperado de: http://archivos.liccom.edu.uy/Figuras/Dewey

Elliott, D. (1995). Music Matters. A New Philosophy of Music Education. New York: Oxford University Press.

Froehlich, H. (2011). Sociología para el profesorado de música. Barcelona: Editorial Graó.

González, J. (2001). Musicología popular en América Latina: síntesis de sus logros, problemas y desafíos. Revista Musical Chilena, 55(195), 38-64. Recuperado de: https://revistamusicalchilena.uchile.cl/index.php/RMCH/article/view/485/398

@Raúl Jorquera Rossel, Ximena Valverde Ocariz y Pere Godall Castell. The content of this article is the sole responsibility of the authors. The Revista Electrónica de LEEME and Universitat de València are not liable for any legal actions that may arise involving the article's content. Revista Electrónica de LEEME - Lista Electrónica Europea de Música en la Educación-. http://ojs.uv.es/index/php/LEEME/index ISSN: 1575-9563. Editores: Universidad de Valencia y Jesús Tejada. Visibilidad de esta revista: SCOPUS, Emerging Sources Citation Index (Clarivate), EBSCO, CINDOC (CSIC), Citefactor, COPAC, Dialnet, DICE (CSIC), DOAJ, e-revistas (CSIC), EBSCO Premier, ERIH+, Gale Cengage Learning, IN-RECS, IRESIE, LATINDEX, MIAR, OCLC Worldcat, RESH, REDIB, RILM Core Journals, SUDOC, ULRICHS. Esta revista es de acceso libre mediante licencia Creative Commons 4.0 CC by. Política de archivado: etiqueta verde SHERPA-ROMEO. 
Herrada, (2016). La educación y los educadores en tiempos Postmodernos. Algunas Reflexiones en Torno a la Educación Musical. Revista Multidisciplinaria Dialógica, 13 (2), 190211. Recuperado de: https://dialnet.unirioja.es/descarga/articulo/6219291.pdf

Jorquera, M. (2010). Modelos didácticos en la enseñanza musical: el caso de la escuela española. Revista Musical Chilena, LXIV(214), 52-74. Recuperado de: https://scielo.conicyt.cl/pdf/rmusic/v64n214/art06.pdf

Jorquera, R. (2019). Músicas populares urbanas en ámbitos de la educación musical chilena. Análisis de su inclusión y exclusión. [Tesis Doctoral no publicada]. Universidad Autónoma de Barcelona, Barcelona.

Jorquera, R. y Godall, P. (2018). Inclusión de música popular urbana por parte de docentes de música en Chile. Revista Neuma, 11(2), 132-159. Recuperado de: http://neuma.utalca.cl/?p=491

Macdonald, A. (2016). Music Education Philosophy from Practical to Praxial. (Thesis of Degree of Bachelor of Artswith Departmental Honors in Music). Wesleyan University. Recuperado de: https://wesscholar.wesleyan.edu/cgi/

McLaren, P. (1990). Prefacio: Teoría crítica y significado de la esperanza. En H.A. Giroux. Los profesores como intelectuales (pp.11-24). Barcelona: Paidós.

Mendivil, J. (2016). En contra de la música. Herramientas para pensar, comprender y vivir las músicas. Buenos Aires: Gourmet Musical Ediciones.

Murillo, A. (2015). ¿Más que música? Natura 3.0: diluyendo las fronteras del arte en las aulas de música de secundaria. En A. Giraldez (Coord.), De los ordenadores a los dispositivos móviles. Propuestas de creación musical y audiovisual (pp.49-68). Barcelona: Editorial Graó.

Pliego, G. (2012). La filosofía praxial en la formación universitaria del profesorado de educación musical. [Tesis Doctoral no publicada]. Universidad de Salamanca, Salamanca.

Polo, M. y Pozzo, M. (2011). La música popular tradicional en el currículum escolar: ¿un aporte a la formación del "ser nacional" o a la educación para la democracia? Contextos Educativos, 14, 191-202. Recuperado de: https://dialnet.unirioja.es/descarga/articulo/

Regelski, T. (2009). La música y la educación musical: Teoría y práctica para "marcar la diferencia”. En D. Lines (Comp.), La educación musical en el nuevo milenio (pp.2147). Madrid: Morata.

@Raúl Jorquera Rossel, Ximena Valverde Ocariz y Pere Godall Castell. The content of this article is the sole responsibility of the authors. The Revista Electrónica de LEEME and Universitat de València are not liable for any legal actions that may arise involving the article's content. Revista Electrónica de LEEME - Lista Electrónica Europea de Música en la Educación-. http://ojs.uv.es/index/php/LEEME/index ISSN: 1575-9563. Editores: Universidad de Valencia y Jesús Tejada. Visibilidad de esta revista: SCOPUS, Emerging Sources Citation Index (Clarivate), EBSCO, CINDOC (CSIC), Citefactor, COPAC, Dialnet, DICE (CSIC), DOAJ, e-revistas (CSIC), EBSCO Premier, ERIH+, Gale Cengage Learning, IN-RECS, IRESIE, LATINDEX, MIAR, OCLC Worldcat, RESH, REDIB, RILM Core Journals, SUDOC, ULRICHS. Esta revista es de acceso libre mediante licencia Creative Commons 4.0 CC by. Política de archivado: etiqueta verde SHERPA-ROMEO. 
Romé, S. (2015). Introducción. En S. Romé (Ed.), Música popular. Epistemología y didáctica en música latinoamericana (pp.7-8). La Plata: Universidad Nacional de La Plata.

Rosabal-Coto, G. (2008). El pragmatismo en educación musical. Sonograma. Revista de pensament musical, 2(6). Recuperado de: http://sonograma.org/num 02/articles/

Shifres, F., Gonnet, D. (2015). Problematizando la herencia colonial en la educación Musical. Epistemus. Revista de estudios en Música, Cognición y Cultura, 3(2). 51-67. Recuperado de: https://revistas.unlp.edu.ar/Epistemus/article/view/2971/2712

Small, C. (1998). Musicking: The Meanings of Performing and Listening. Middletown, Connecticut: Wesleyan University Press.

Uribe, C. (2010). La educación musical hoy: ¿cita a ciegas o cambio de paradigma? Revista Neuma, Año 3(1), 104-120. Recuperado de: https://neuma.utalca.cl//wpcontent/uploads/2018/03/Vol_2010_articulo-6.pdf

Valverde, X. (2018). Música tradicional en el aula: las bandas de bronces de Tarapacá y sus aportaciones a la educación musical escolar. [Tesis Doctoral no publicada]. Universidad Autónoma de Barcelona, Barcelona.

@Raúl Jorquera Rossel, Ximena Valverde Ocariz y Pere Godall Castell. The content of this article is the sole responsibility of the authors. The Revista Electrónica de LEEME and Universitat de València are not liable for any legal actions that may arise involving the article's content. Revista Electrónica de LEEME - Lista Electrónica Europea de Música en la Educación-. http://ojs.uv.es/index/php/LEEME/index ISSN: 1575-9563. Editores: Universidad de Valencia y Jesús Tejada. Visibilidad de esta revista: SCOPUS, Emerging Sources Citation Index (Clarivate), EBSCO, CINDOC (CSIC), Citefactor, COPAC, Dialnet, DICE (CSIC), DOAJ, e-revistas (CSIC), EBSCO Premier, ERIH+, Gale Cengage Learning, IN-RECS, IRESIE, LATINDEX, MIAR, OCLC Worldcat, RESH, REDIB, RILM Core Journals, SUDOC, ULRICHS. Esta revista es de acceso libre mediante licencia Creative Commons 4.0 CC by. Política de archivado: etiqueta verde SHERPA-ROMEO. 